\title{
Multidimensional Texture Analysis for Improved Prediction of Ultrasound Liver Tumor Response to Chemotherapy Treatment
}

\author{
Omar S. Al-Kadi ${ }^{1,2(凶)}$, Dimitri Van De Ville ${ }^{2,3}$, and Adrien Depeursinge $e^{2,4}$ \\ 1 King Abdullah II School for Information Technology, \\ University of Jordan, Amman, Jordan \\ 2 School of Engineering, École Polytechnique Fédérale de Lausanne (EPFL), \\ Lausanne, Switzerland \\ omar.alkadi@epfl.ch \\ 3 Department of Radiology and Medical Informatics, \\ Université de Genève, Geneva, Switzerland \\ ${ }^{4}$ University of Applied Sciences Western Switzerland (HES-SO), Sierre, Switzerland
}

\begin{abstract}
The number density of scatterers in tumor tissue contribute to a heterogeneous ultrasound speckle pattern that can be difficult to discern by visual observation. Such tumor stochastic behavior becomes even more challenging if the tumor texture heterogeneity itself is investigated for changes related to response to chemotherapy treatment. Here we define a new tumor texture heterogeneity model for evaluating response to treatment. The characterization of the speckle patterns is performed via state-of-the-art multi-orientation and multi-scale circular harmonic wavelet $(\mathrm{CHW})$ frames analysis of the envelope of the radio-frequency signal. The lacunarity measure - corresponding to scatterer number density - is then derived from fractal dimension texture maps within the CHW decomposition, leading to a localized quantitative assessment of tumor texture heterogeneity. Results indicate that evaluating tumor heterogeneity in a multidimensional texture analysis approach could potentially impact on designing an early and effective chemotherapy treatment.
\end{abstract}

\section{Introduction}

Liver tumor ultrasound scanning is recently becoming increasingly recommended as a first diagnosis option for early prediction of response to chemotherapy treatment [1]. However, visual assessment of tumor response to chemotherapy is very challenging without monitoring longitudinally the tumor development. This is, in part, due to the intertwined tumor speckle variations, leading to formation of complex texture patterns. A robust approach to tackle this texture complexity is to assess the radio-frequency ( $\mathrm{RF}$ ) echoes - instead of B-mode images - which are not subjected to log-compression and proprietary filtering algorithms. This original data preservation allows for better statistical modeling of backscattering properties.

(C) Springer International Publishing AG 2016

S. Ourselin et al. (Eds.): MICCAI 2016, Part I, LNCS 9900, pp. 619-626, 2016

DOI: $10.1007 / 978-3-319-46720-7 \_72$ 
During the course of chemotherapy treatment, changes within tumor region may occur due to progression or regression of disease. The speckle patterns variations are heterogeneous as tumor angiogenesis can affect the complexity of the tissue spatial relationship. This heterogeneity in tumor tissue scatterers could span different scatterer densities. Regions within the tumor tissue that respond to treatment exhibit different statistical properties to that of the non-respondent counterpart [2-4,6]. Thus it is essential to improve the discriminative abilities of the employed statistical distribution model for better characterization of tumor heterogeneity.

A number of contributions employed the backscattered statistics from RF ultrasound signal for evaluating the early death of tumor cells in response to chemotherapy treatment; such as using the scatterer spacing and diameter, and acoustic concentration in combination with texture features from the gray-level co-occurrence matrix [3]. Others relied on calculating the power spectrum from the Fourier transform of raw RF data, and subsequently deriving the spectral slope, $0-\mathrm{MHz}$ intercept, and mid-band fit quantitative parameters [2]. In another similar work, the maximum mean discrepancy features were extracted on histograms of quantitative ultrasound spectroscopic parametric maps [4]. However, analyzing tissue backscattering properties from a single resolution perspective is limiting, as substantial information that could assist in tumor tissue characterization can be hidden at different locations, orientations and scales of resolution. Refined statistical properties can be obtained from the RF envelope-detected signal performed in 2D [5], where a fractal-based representation that underpins a tumor model can be achieved [6].

For an improved ultrasound tissue characterization of tumor texture, the use of the fractal dimension (FD) as in [6] might not capture well all relevant tissue changes. Namely, the FD corresponds to scatterers spatial distribution and not scatterer number density, thus it is possible to get similar FD values for textures which might not look alike. As a result, this could overlook some of the important aspects of the statistics of the envelope detected from the RF signal. Tissue heterogeneity property previously proved to be useful in assessing tumor aggressiveness [7]. Therefore, the variation in scatterer number density within the tumor focal region - which corresponds to spatial heterogeneity - would be an interesting property to consider as well.

Here we propose a novel ultrasound texture analysis approach for tissue characterization. The assumption is made that quantifying tumor spatial heterogeneity could assist in revealing subtle cues (i.e., small changes in tissue texture) for tumors that responded to chemotherapy administration. A Nakagami statistical distribution is fitted locally to the envelope RF signal for estimating the backscattering parameters. Subsequently, circular harmonic wavelets frames are used to decompose the backscattered shape statistics into different spatial scales and local circular frequencies. Finally, a heterogeneity feature descriptor is successively constructed by mapping the circular harmonic wavelets frames on the fractal dimension space, and then quantitatively estimating tissue sparsity from the constructed fractal texture maps. 


\section{Materials and Methods}

Although the backscattered signal from tumor tissue tends to show a stochastic pattern, the local concentration and spatial arrangement of progressive tumor tissue scatterers may follow a distribution different from regressive ones. To maximize the difference between the two conditions, the fractal signatures are derived from multi-scale circular frequency analysis of the acoustic properties of the envelope RF signal for assessing tissue heterogeneity.

\subsection{Ultrasound RF Data Analysis}

The amplitudes of the individual backscattered signals are assumed to be randomly distributed due to the random backscatter coefficient of each individual scatterer. The interference signals from the large number of randomly distributed scatterers give the echo signal its statistical nature. Many statistical models exist for the purpose of characterizing randomness in soft tissue; however, very few can estimate the model parameters with analytical simplicity and computational ease. The Nakagami distribution is an example of a simple bi-parametric model which can characterize tissue in various scattering conditions and scatterer densities [8]. The Nakagami density function is defined as

$$
P_{n}(x \mid m, \Omega)=\frac{2 m^{m}}{\Gamma(m) \Omega^{m}} x^{2 m-1} e^{-m x^{2} / \Omega},
$$

for $x \geq 0$, where $\Gamma$ is the Euler gamma function. The real numbers $m>0$ (related to the local concentration of scatterers) and $\Omega>0$ (related to the local backscattered energy) are called the shape and scaling parameters, respectively. Similarly to the Rayleigh distribution, the envelope of the RF signal $x^{2}$ follows a gamma distribution. By fine-tuning the shape of the distribution parameter $m$, other statistical distributions can be modeled, such as, an approximation of the Rician distribution (i.e., post-Rayleigh) for $m>1$, a Rayleigh distribution for the special case when $m=1$, and when $m<1$ a K-distribution (i.e., preRayleigh). The envelope-detected RF signal based on the Nakagami $m$ parameter was used subsequently for investigating tissue heterogeneity.

\subsection{Circular Harmonic Wavelets}

A natural way of assessing the echo signal $f(x, y)$ is to analyse its statistical properties at different spatial scales. An efficient way to systematically decompose $f(x, y)$ into successive dyadic scales is to use a redundant isotropic wavelet transform. The recursive projection of $f$ on Simoncelli's isotropic wavelet provides such a frame representation [9]. The radial profile of the wavelet function is defined in polar coordinates in the Fourier domain as

$$
\hat{h}(\rho)= \begin{cases}\cos \left(\frac{\pi}{2} \log _{2}\left(\frac{2 \rho}{\pi}\right)\right), & \frac{\pi}{4}<\rho \leq \pi \\ 0, & \text { otherwise. }\end{cases}
$$


The scaling function is not used to ensure illumination invariance. The local structural properties of $f(x, y)$ can be well described in terms of the local circular frequencies, which was at the origin of the success of methods such as local binary patterns (LBP) [10]. In this work, local circular harmonics are computed on top of the wavelet frames to characterize circular frequencies at multiple scales [11], which is an extension of steerable Riesz wavelets [12]. Circular harmonic wavelets (CHW) of order $n$ are constructed in the Fourier domain as

$$
\hat{\phi}^{(n)}(\rho, \varphi)=\hat{h}(\rho) \mathrm{e}^{\mathrm{j} n \varphi} .
$$

The representation obtained from the collection of the complex magnitudes of the scalar products $\left|\left\langle f, \phi^{(n)}\right\rangle\right|$ characterizes the local circular frequencies in $f(x, y)$ up to an order $n=1: N$ and is rotation invariant [13].

\subsection{Heterogeneity Quantification}

After the projection of $f$ on $\mathrm{CHW}$ frames, the self-similarity of each voxel from its surrounding neighborhood is determined via estimating its localized FD. This would serve as an estimated array of localized FD values (i.e. fractal texture map) for a multi-dimensional representation of tissue heterogeneity.

Fractal Texture Map Estimation. There are several methods to estimate the FD, however multiplicative speckle scale changes can effect the stability of parameter estimation. The fractal Brownian motion (fBm), which is known for its capability for describing random phenomena [14], can work well with ultrasound tissue characterization [6]. Both scale- and rotation-invariance properties of the non-stationary fBm model makes it a perfect candidate to be integrated with the Nakagami modeling and CHW decomposition. The fBm can be characterized by

$$
E(\Delta v)=K \Delta r^{H},
$$

where $E(\Delta v)=|q-p|$ is the mean absolute difference of voxel pairs $\Delta v ; \Delta r=$ $\sqrt{\sum_{i=1}^{s}(q-p)^{2}}(s=3$ for fBm texture surface enveloped in $3-\mathrm{D}$ space $)$ is the voxel pair distances; $H$ is the Hurst exponent; and $K>0$ is a constant.

Given a volume set $V$ of constructed envelope-detected RF tumor regions $f_{i}^{\mu}(x, y)$, where $\mu$ stands for the Nakagami shape parameter and $i$ is a certain slice in the acquired volume, tissue fractal characteristics from the backscattered envelope are investigated. A fractal texture map $\mathcal{F}$, having a size $m \times n$ and for $k$ dimensions, can be defined as in (5) based on the CHW frames for all corresponding voxels $v_{x y}^{k}$ of $f_{i}^{\mu}(x, y), f \in V$. The $k$ value empirically specifies the maximum convolution kernel size $I$ used in estimating $\Delta v$ of (4). The slope of linear regression line of the log-log plot of (4) gives $H$ from which the localized fractal dimension is estimated $(F D=3-H)$. This procedure is iterated covering all $v_{x y}^{k}$ which yields a set of multi-dimensional fractal texture maps $\mathcal{M}_{f}$ to be constructed for each $V$, where $\mathcal{M}_{f}=\left\{\mathcal{F}_{1}, \mathcal{F}_{2}, \ldots, \mathcal{F}_{z}\right\}$, and $z$ is the total number of $\mathcal{F}$ in $\mathcal{M}_{f}$. 


$$
\mathcal{F}^{(N, J)}\{f\}(x, y)=\left(\begin{array}{llllll}
v_{11}^{k} & v_{12}^{k} & \cdots & v_{1 y}^{k} & \cdots & v_{1 n}^{k} \\
v_{21}^{k} & v_{22}^{k} & \cdots & v_{2 y}^{k} & \cdots & v_{2 n}^{k} \\
\vdots & \vdots & \ddots & \vdots & \vdots \\
v_{x 1}^{k} & v_{x 2}^{k} & \cdots & v_{x y}^{k} & \cdots & v_{x n}^{k} \\
\vdots & \vdots & & \vdots & \ddots & \vdots \\
v_{m 1}^{k} & v_{m 2}^{k} & \cdots & v_{m y}^{k} & \cdots & v_{m n}^{k}
\end{array}\right)
$$

The integration of the $\mathrm{fBm}$ model at different $\mathrm{CHW}$ orders $N$ and scales $J$ can contribute towards a better separability between the mixtures of speckle patterns within tissue. Such that $\Delta v$ and $\Delta r$ locally estimate the FD of each $v_{x y}^{k}$ up to the resolution limits of $f_{i}^{\mu}$ specified by $k$ that best characterizes the speckle patterns for different scales and circular frequencies. This approach enables for further probing the resolution of CHW frames, and hence facilitates for assessing the speckle pattern heterogeneity.

Lacunarity Analysis. To further differentiate between textures having similar FD values, the lacunarity $(\mathcal{L})$ - a coefficient of variation that can measure the sparsity of the fractal texture - can assist in quantifying aspects of patterns that exhibit scale-dependent changes in structure [15]. Namely, it measures the heterogeneity of the fractal pattern, providing meta-information about the dimension of $\mathcal{F}$. The lower the $\mathcal{L}$ value, the more heterogeneous the examined tumor region $f_{i}^{\mu}$ represented by $\mathcal{F}$, and vice versa. $\mathcal{L}$ can be defined in terms of the relative variance of the size distribution in $\mathcal{F}$ as

$$
\mathcal{L}=\frac{\frac{1}{m n} \sum_{x} \sum_{y} \mathcal{F}^{2}-\left(\frac{1}{m n} \sum_{x} \sum_{y} \mathcal{F}\right)^{2}}{\left(\frac{1}{m n} \sum_{x} \sum_{y} \mathcal{F}\right)^{2}}=\frac{E\left[\mathcal{F}^{2}\right]-E[\mathcal{F}]^{2}}{E[\mathcal{F}]^{2}}=\frac{\operatorname{Var}[\mathcal{F}]}{E[\mathcal{F}]^{2}} .
$$

\section{Results and Discussion}

\subsection{Clinical Tumor Cross-Sectional Dataset}

The approach has been validated on RF ultrasound data acquired using a diagnostic ultrasound system (Zonare Medical Systems, Mountain View, CA, USA) $4 \mathrm{MHz}$ curvilinear transducer and $11 \mathrm{MHz}$ sampling. The output 2-D image size was $65 \times 160 \mathrm{~mm}$ with a resolution of $225 \times 968$ pixels. A total of 287 cross sectional images of 33 volumetric liver tumors manually segmented were obtained from the stacks of 2-D images, 117 were responsive and 170 did not responded to chemotherapy treatment. Response to treatment was determined based on conventional computed tomography follow up imaging as part of the patient standard clinical care based on the response evaluation criteria in solid tumors (RECIST) [16]. The baseline cross-sectional imaging was compared against those performed at the end of treatment according to the RECIST criteria to determine response to treatment for each target tumor. A tumor was classified as responsive if categorized as partial response and non-responsive if no change or disease demonstrated progression. 


\subsection{Statistical Analysis}

To quantitatively assess the robustness of our approach, $2(N+1) \times J$ features, where 2 stands for both the average $\mathcal{F}$ and $\mathcal{L}$ estimated at each $N$ and $J$ per slice of each of the acquired volumes, were fed into a support vector machine classifier to compare the overall modeling performance of classifying responsive versus non-responsive cases. Cross-validation was performed based on a leaveone-tumor-out (loo) approach, and further validated on independent test-set of 107 cross sectional images (69 responsive versus 38 non-responsive images). The convolution kernel size $I$ used in estimating the localized FD of $\mathcal{F}$ was initially optimized while having $N$ and $J$ fixed, see Fig. 1 . Then the classification performance for different $N$ and $J$ values of the $\mathrm{CHW}$ representation was investigated in order to quantify $\mathcal{L}$ extracted from $\mathcal{F}$. Hence, when the optimized values of $N$, $J$ and $I$ are employed, $97.91 \%$ (97.2\% for unseen data) best classification accuracy is achieved as compared to $92.1 \%$ in the work of [6], and similarly applies to the 5- and 10-folds cross validation results (indicated in terms of mean \pm standard deviation of the performance over 60 runs). Figure 2 shows the $\mathcal{F}$ and corresponding $\mathcal{L}$ for a non-responsive vs responsive case. A less heterogeneous texture (i.e. higher $\mathcal{L}$ values colored in red in Fig. 2) is witnessed in the responsive case. This indicates tumor tissue texture is becoming more sparse, which could be signs of necrotic regions, and hence responding to treatment (Table 1).

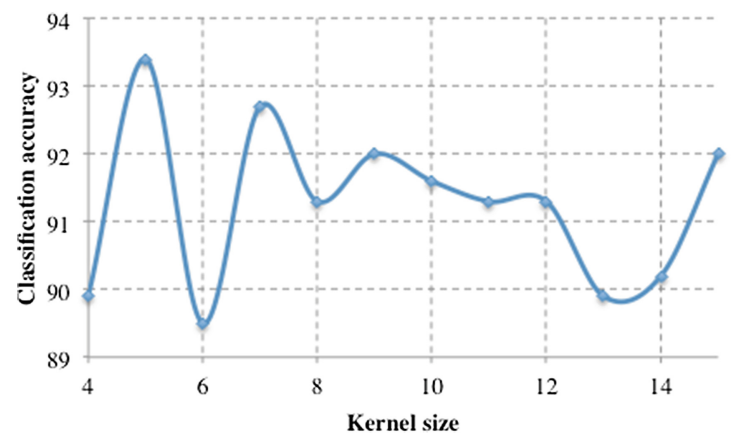

Fig. 1. Classification accuracies for varying convolution kernel size $(I)$ in pixels with fixed order $(N=2)$ and scale $(J=6)$

Characterizing the speckle patterns in terms of multi-scale circular harmonics representation could assist in better characterization of the backscattered signal, which adapts according to the varying nature of tissue structure. As changes in the scatterers' spatial distribution and number density reflect in the ultrasound backscattering, the sensitivity of response to treatment of the envelope RF signal is implicitly linked to changes in FD and associated $\mathcal{L}$ on the $\mathrm{CHW}$ frames. Finally, tumors with varying depth would decrease the amplitude of the RF data, and quantifying the tumor response to chemotherapy treatment under such conditions is planned for future work. 
Table 1. Classification performance for the multidimensional heterogeneity analysis of clinical liver tumor dataset

\begin{tabular}{l|l|l|l}
\hline \multirow{2}{*}{ Statistical measures } & \multicolumn{3}{l}{ Cross-validation } \\
\cline { 2 - 4 } & loo & 5-fold & 10-fold \\
\hline Accuracy & 97.91 & $93.30 \pm 0.017$ & $95.70 \pm 0.009$ \\
\hline Sensitivity & 98.80 & $96.40 \pm 0.888$ & $97.50 \pm 0.931$ \\
\hline Specificity & 96.60 & $88.80 \pm 0.964$ & $93.10 \pm 0.975$ \\
\hline ROC-AUC & 97.70 & $92.60 \pm 0.020$ & $95.30 \pm 0.009$ \\
\hline
\end{tabular}

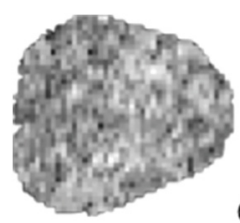

(a)

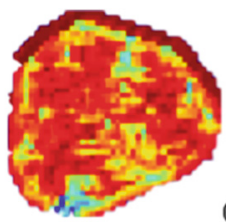

(b)

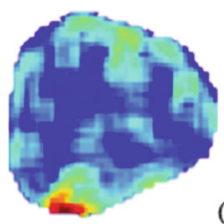

(c)

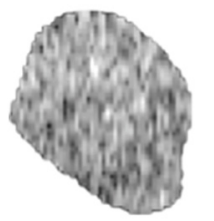

(d)

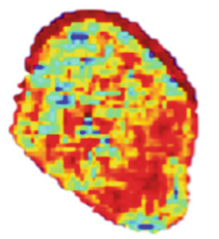

(e)

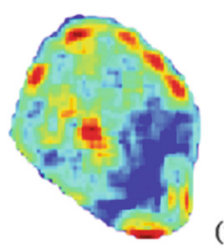

Fig. 2. (1st column) Tumor B-mode images, (2nd column) fractal texture maps and (3rd column) corresponding tissue heterogeneity representation for a (1st row) nonresponsive vs (2nd row) responsive case, respectively. Red regions in (c) and (f) indicate response to treatment according to RECIST criteria [16]. CHW decomposition was based on a 2 nd order and up to the 8 -th scale.

\section{Conclusion}

A novel approach has been presented for quantifying liver tumor response to chemotherapy treatment with three main contributions: (a) ultrasound liver tumor texture analysis based on a Nakagami distribution model for analyzing the envelope RF data is important to retain enough information; (b) a set of CHW frames are used to define a new tumor heterogeneity descriptor that is characterized at multi-scale circular harmonics of the ultrasound RF envelope data; (c) the heterogeneity is specified by the lacunarity measure, which is viewed as the size distribution of gaps on the fractal texture of the decomposed CHW coefficients. Finally the measurement of heterogeneity for the proposed representation model is realized by means of support vector machines.

Acknowledgments. We would like to thank Dr. Daniel Y.F. Chung for providing the ultrasound dataset. This work was partially supported by the Swiss National Science Foundation (grant PZ00P2_154891) and the Arab Fund (grant 2015-02-00627). 


\section{References}

1. Bae, Y.H., Mrsny, R., Park, K.: Cancer Targeted Drug Delivery: An Elusive Dream, pp. 689-707. Springer, New York (2013)

2. Sadeghi-Naini, A., Papanicolau, N., Falou, O., Zubovits, J., Dent, R., Verma, S., Trudeau, M., Boileau, J.F., Spayne, J., Iradji, S., Sofroni, E., Lee, J., Lemon-Wong, S., Yaffe, M., Kolios, M.C., Czarnota, G.J.: Quantitative ultrasound evaluation of tumor cell death response in locally advanced breast cancer patients receiving chemotherapy. Clin. Cancer Res. 19(8), 2163-2174 (2013)

3. Tadayyon, H., Sadeghi-Naini, A., Wirtzfeld, L., Wright, F.C., Czarnota, G.: Quantitative ultrasound characterization of locally advanced breast cancer by estimation of its scatterer properties. Med. Phys. 41, 012903 (2014)

4. Gangeh, M.J., Sadeghi-Naini, A., Diu, M., Kamel, M.S., Czarnota, G.J.: Categorizing extent of tumour cell death response to cancer therapy using quantitative ultrasound spectroscopy and maximum mean discrepancy. IEEE Trans. Med. Imaging 33(6), 268-272 (2014)

5. Wachinger, C., Klein, T., Navab, N.: The 2D analytic signal for envelope detection and feature extraction on ultrasound images. Med. Image Anal. 16(6), 1073-1084 (2012)

6. Al-Kadi, O.S., Chung, D.Y., Carlisle, R.C., Coussios, C.C., Noble, J.A.: Quantification of ultrasonic texture intra-heterogeneity via volumetric stochastic modeling for tissue characterization. Med. Image Anal. 21(1), 59-71 (2015)

7. Al-Kadi, O.S., Watson, D.: Texture analysis of aggressive and non-aggressive lung tumor CE CT images. IEEE Trans. Bio-med. Eng. 55(7), 1822-1830 (2008)

8. Shankar, P.M.: A general statistical model for ultrasonic backscattering from tissues. IEEE T Ultrason. Ferroelectr. Freq. Control 47(3), 727-736 (2000)

9. Portilla, J., Simoncelli, E.P.: A parametric texture model based on joint statistics of complex wavelet coefficients. Int. J. Comput. Vis. 40, 49-70 (2000)

10. Ojala, T., Pietikänen, M., Mäenpää, T.: Multiresolution gray-scale and rotation invariant texture classification with local binary patterns. IEEE Trans. Pattern Anal. Mach. Intell. 24, 971-987 (2002)

11. Unser, M., Chenouard, N.: A unifying parametric framework for $2 \mathrm{D}$ steerable wavelet transforms. SIAM J. Imaging Sci. 6(1), 102-135 (2013)

12. Unser, M., Van De Ville, D.: Wavelet steerability and the higher-order Riesz transform. IEEE Trans. Image Process. 19(3), 636-652 (2010)

13. Depeursinge, A., Püspöki, Z., et al.: Steerable wavelet machines (SWM): learning moving frames for texture classification. IEEE Trans. Image Process. (submitted)

14. Lopes, R., Betrouni, N.: Fractal and multifractal analysis: a review. Med. Image Anal. 13(4), 634-649 (2009)

15. Plotnick, R.E., Gardner, R.H., Hargrove, W.W., Prestegaard, K., Perlmutter, M.: Lacunarity analysis: a general technique for the analysis of spatial patterns. Phys. Rev. E 53(5), 5461-5468 (1996)

16. Eisenhauer, E.A., Therasse, P., et al.: New response evaluation criteria in solid tumours: revised RECIST guideline. Eur. J. Cancer 45(2), 228-247 (2009) 